\title{
Development of Measurement Equipment for Calibrating Beam Position Monitor in HLS Storage Ring
}

\author{
Lianguan Shen, Department of Precision Machinery \& Precision Instrumentation \\ Junhua Wang, Guicheng Wang, National Synchrotron Radiation Laboratory \\ Xin He, Jianbin Zhao, Yangbin Zhu, Jianping Yao, Department of Precision Machinery \& \\ Precision Instrumentation \\ University of Science \& Technology of China \\ Hefei, Anhui, 230026 China
}

\begin{abstract}
An automatic calibration system for BPM (Beam Position Monitor) of HLS (Hefei Synchrotron Radiation Light Source) storage ring is described in this paper. The system integrated a manipulator for holding simulated antenna vertically to move in horizontal plane, a signal collector and a data processor. The positioning accuracy of antenna, thin wire, is monitored by a set of microscopes with reading resolution of $0.1 \mu \mathrm{m}$. Optical gratings with veracity of $5 \mu \mathrm{m}$ obtain the position of the antenna. The system has been used for the 2 nd phase of HLS to calibrate the BPM detectors and measured 4 vacuum chambers, which have been installed in the storage ring. Testing results show that the performance of the system is good enough for calibration.
\end{abstract}

\section{INTRODUCTION}

The beam position monitors are necessary for observing transverse position of beam in storage ring. Usually they should be calibrated before installation to understand the relation between spatial electronic field and the induced voltage. The whole experimental system is shown in Fig.1. In it the beam is simulated by means of a thin wire antenna.

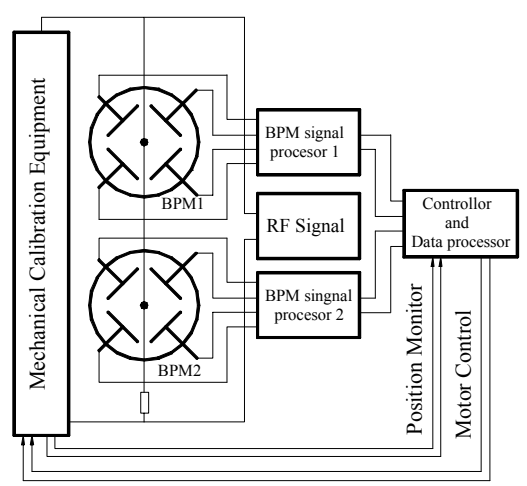

Fig.1 Frame of the whole experimental system

In order to get a high accurate result of the whole experimental system, the mechanical accuracy of the mechanical equipment is the key issue. We should guarantee that the position of the wire can be measured exactly and the positional accuracy both at initial position and sampling points should be high during mapping. Actually, the focus of the issue is to build a mechanical equipment of high precision.

\section{THE MECHANICAL EQUIPMENT}

The long flexible thin wire should be pinpointed regardless of initial or moving. It should be straight and move on an even keel. The wire can lie horizontally or vertically. The movement parts can be wire holder or vacuum chamber. We select the in horizontal plane to avoid too large tensile force to make wire straight. Furthermore, considering the eccentric chamber structure, we move the wire carriage to increase the stability.

The mechanical equipment is composed of machine with NC worktable, wire holder, tensile mechanism, micro adjustor of wire and guider for installation of chamber. Fig. 2 is the drawing of the equipment.

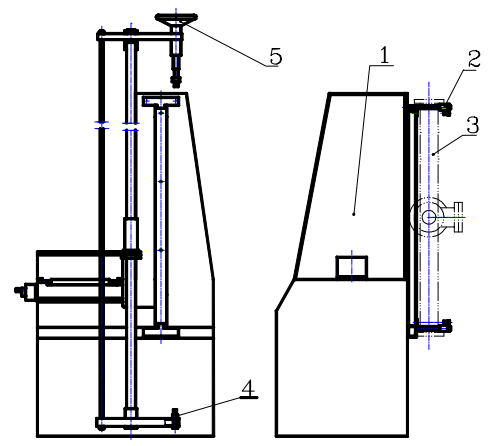

Fig.2 The mechanical equipment

Here, 1 is the machine bed. 2 is the clamper for installing vacuum chamber. 3 is the measured chamber. 4 is the micro adjuster and 5 is the tensile wheel.

\subsection{Machine base}

To obtain high performance and low cost, we choose the bed of a ready-made electric-discharge machine as the base of machine. There are two axes with stepping motor which step resolution is $1 \mu \mathrm{m}$ and no creeping. We only need to attach two gratings on it to detect the position of the measuring points. The veracity of $5 \mu \mathrm{m}$ of gratings was measured by manufacturer beforehand and can be used for modify measurement result if it is necessary. 


\subsection{Clamping of the chamber}

Every measurement of chamber must have uniform benchmark. High installing repeatability of chambers is necessary. Therefore an appropriate structure as follows is designed. With it the installing repeatability can be controlled within $10 \mu \mathrm{m}$. The two parallel columns of the clamp have been adjusted to parallel to the $\mathrm{Y}$-axis of the worktable. The parallelism is less than $10 \mu \mathrm{m}$. This makes mapping measurement high accuracy.

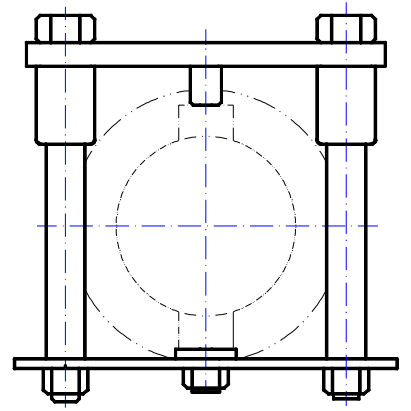

Fig. 3 Clamp mechanism

\subsection{Tensile structure}

The simulated antenna should be hauled. The tensile mechanism must be satisfied for the following exigent demands. Such as: 1 just right tensile force. It should be neither too large to make the wire plastic yield, nor too small to loosen the wire. 2 its position offset from datum line should be as small as possible. 3 Easy to finetuning. Fig. 4 shows the clamp structure. A positional repeatability of $10 \mu \mathrm{m}$ has been obtained.

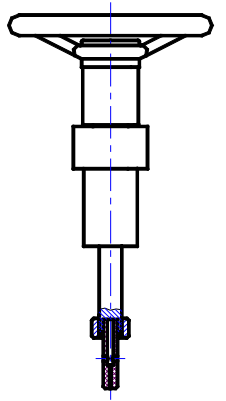

Fig.4 Tensile structure

\section{POSITION MEASUREMENT}

Actually, for every operating the positioning accuracy of measuring points is mainly affected by two factors. One is the initial position of the antenna. Another one is the accuracy and reading error of gratings. Therefore, Adjusting the axis of the wire to be exact consistent with the installing reference mark is the first and foremost. Obviously any contact-type method is invalid here. Experiments with universal optical measure were also in fail for a thin columned wire.
After comparing several microscopes with each other, we found a sort of reading microscope quite suitable. Its reading resolution is $0.1 \mu \mathrm{m}$ and the measurable field is wider than $1 \mathrm{~mm}$. We create a ball head pin of $\phi 1 \mathrm{~mm}$ as reference mark and insert it into datum hole, then drive the wire approaching the pin until the axis of wire coincide with the symmetrical axis of the pin in $\mathrm{X}$ direction and a certain distance from the pin in $\mathrm{Y}$ direction individually. The principle is shown in Fig.5. In such way, a high repeatability of the initial position of wire is obtained.

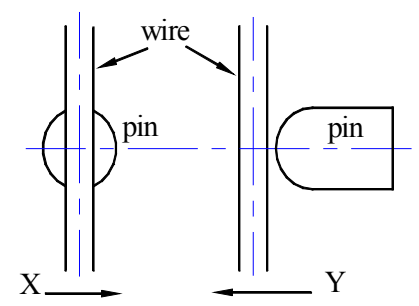

Fig.5 Determination of initial position of wire

\section{SYSTEM INTERGRATION}

We hope the system is of high security and compact. The integrated software packages complete all the actions of the system. Before measuring every chamber, the machine searches the origin point of grating automatically.

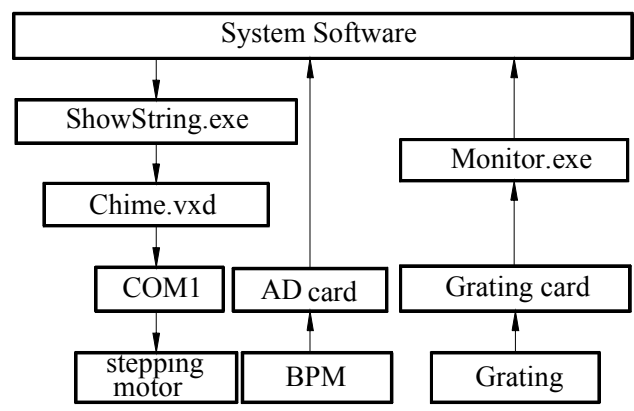

Fig.6 Frame of system software

When the antenna mappings point to point automatically or manually, the data from BPM and grating cards are acquired and recorded. The work status shows on the screen simultaneously. On the bed there isn't any hard limitation of length of path. The program realizes the security. Fig. 6 is the frame of system software.

As a matter of convenience, we design the interface as a virtual control board of instrument. In this way, the only thing for users is to simply click the buttons if they want to do some operations. Fig.7 shows one of interface of the system.

The program also prepared a drawing function. User can evaluate the result on spot, so that some occasional operational error can be duly found. Fig. 8 is the computed result by least square method. 


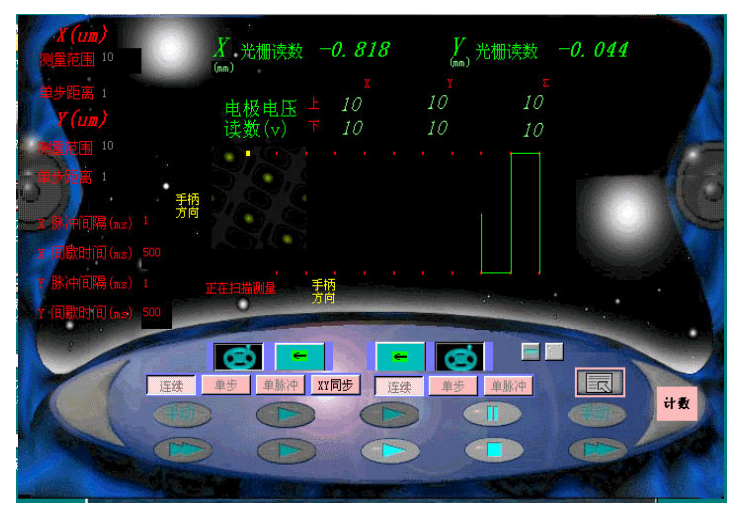

Fig.7 Machine is working

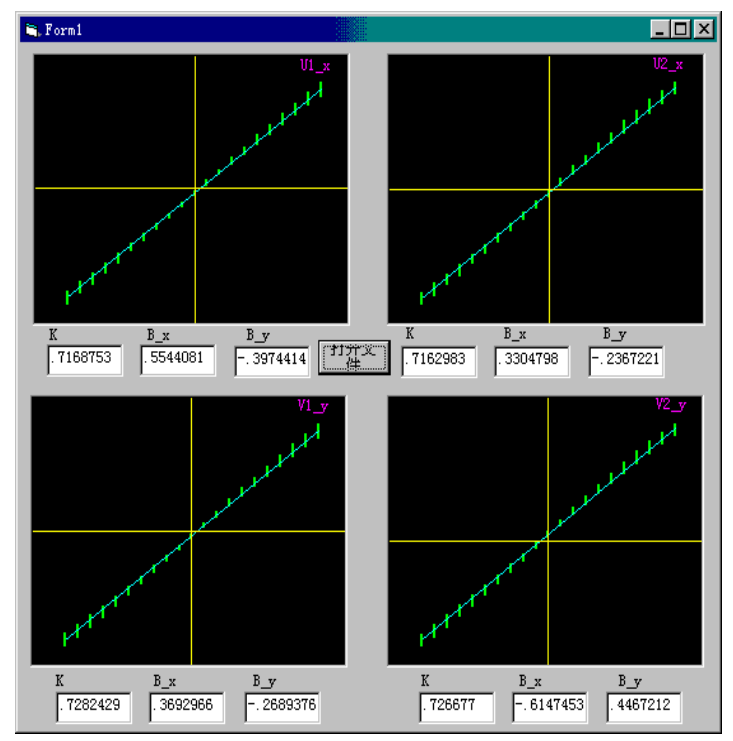

Fig.8 Evaluating the measured result

This system has been used for calibrating 4 vacuum chambers. The system accuracy of the whole experimental system is $\pm 15 \mu \mathrm{m}$. According to the measured data, the $4 \#$ vacuum chamber has been eliminated because its offset from electronic centre to geometric centre in $\mathrm{X}$ direction is as big as $1.578 \mathrm{~mm}$. The mechanical engineer counterchecked its geometrical parameters again. The fact has proved that this conclusion is well adjusted. The remains have been installed into Storage ring. As an example, Fig.9 shows the 3\# vacuum chamber's mapping picture. Its centre offset between electronic and geometric is given in table 1.

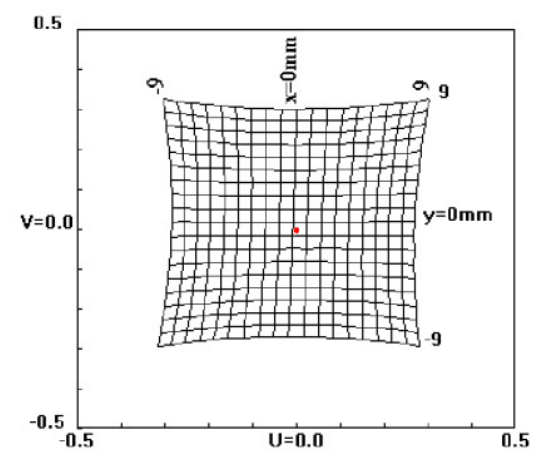

Fig.9 Mapping of measured chamber

Table 1. Offset of center between electric and geometric

\begin{tabular}{|c|c|c|c|}
\hline \multicolumn{2}{|c|}{ Electrode group } & $\mathrm{X}(\mathrm{mm})$ & $\mathrm{Y}(\mathrm{mm})$ \\
\hline \multirow{2}{*}{$3 \#$} & Upside & -0.475767 & 0.193096 \\
\cline { 2 - 4 } & Underside & -0.310661 & -0.478395 \\
\hline
\end{tabular}

\section{REFERENCE}

[1] Junhua Wang, Lianguan Shen, Guicheng Wang, Calibration of BPM Detector and Error analysis in HLS, J. High Energy \& Nuclear Physics, 2001(to be published). 